\title{
Pediatric Meningosarcoma: Clinical Evolution and Genetic Instability
}

Reyes López de Mesa, MD*, Luis Sierrasesúmaga, MD, PhD*, Adela López de Cerain, $\mathrm{PhD \dagger}$, María José Calasanz, PhDł, and Ana PatiñoGarcía, $\mathrm{PhD*}$

From *Laboratory and Department of Pediatrics, †Laboratory of Toxicology, $\ddagger$ Department of Genetics, University Clinic and University of Navarra, Pamplona, Spain.

Communications should be addressed to:

Dr. Patiño-García

Laboratory of Pediatrics; "Los Castaños” Building; University of Navarra; E31080 Pamplona, Spain.

\begin{abstract}
This report presents a female diagnosed with a frontoparietal interhemispheric meningosarcoma who, parallel to the clinical worsening, revealed an increase in the genetic instability (in bleomycin cultures) and the complexity of the karyotypes, with the acquisition of a clonal deletion of 17p13 (the locus for the TP53 tumor suppressor gene). The genetic findings of this patient suggest that the increased genetic instability could contribute to tumor progression as well as to treatment resistance, possibly in the background of the clonal deletion of TP53.
\end{abstract}




\section{INTRODUCTION}

Meningeal sarcomas are tumors arising from mesenchymal and nonmeningothelial cells which account for less than 3\% of all pediatric central nervous system tumors. These tumors are slightly more frequent in the second decade of life, are highly aggressive, and rarely respond to conventional antitumoral treatment [1]. In the past, meningeal sarcomas were classified as a subgroup of aggressive meningeal tumors, although they are nowadays excluded from that group [2] on the basis that the cells of origin are different, which can account for their distinct histopathologic and cytogenetic features.

Several reports have characterized the most frequent chromosomal aberrations that are present in meningiomas. These aberrations are generally related to the aggressiveness or progression of the tumors (anaplastic malignant meningiomas) and include monosomy or deletion of chromosome 22 (NF2 locus), complete or partial loss of chromosome 1, and alterations affecting $10 \mathrm{q}, 14 \mathrm{q}$, and 9q [3-4]. In contrast, these particular alterations are not evident in the meningosarcomas, whose genetic alterations are heterogeneous and to date largely undefined.

The patient reported herein was submitted to our laboratory for examination because she was included in an investigation protocol aimed at analyzing the genetic instability induced by antitumoral regimens in pediatric patients [5].

\section{MATERIALS AND METHODS}

Informed consent was obtained from the affected female and her parents, and the ethical approval was granted by the Ethics Committee of the University Clinic, Pamplona, Spain.

Chromosomal studies were performed on peripheral blood samples following standard procedures. A minimum of 50 well-spread metaphases, obtained from 72-hour phytohemagglutinin-stimulated cultures, were analyzed. Chromosomal abnormalities, identified by G-banding, were described according to the international system for human cytogenetic nomenclature [6].

Bleomycin cultures were established as previously described [5]. Breaks were scored in 50 metaphases per sample, and the number of breaks per cell (b/c index) was averaged. Only chromatid breaks or exchanges were recorded; chromatid gaps or attenuated regions were disregarded. A b/c index above 0.8 was considered to indicate sensitivity to bleomycin-induced chromosome damage, and an index above 1.0 was considered to indicate hypersensitivity.

\section{CASE REPORT}

The patient was a 9-year-old female with antecedents of cephalgia and unspecified visual alterations for 2 months. The computed tomographic scan revealed a frontoparietal interhemispheric lesion which was compatible with a meningioma. 
Complete macroscopic resection was achieved, with an inconclusive pathologic diagnosis of meningeal tumor with moderate atypia and areas of cortical infiltration. Within 2 months, the patient relapsed, and the new tumor mass was incompletely resected because of cortical infiltration. Microscopic examination revealed a mesenchymal neoplasia suggestive of meningosarcoma. One month later, complete macroscopic resection of the infiltrating lesion of the medial line was again performed, tumoral areas were more differentiated, there were atypias and carcinomatosis. With the diagnosis of relapsed meningeal sarcoma, she was admitted to the Clínica Universitaria, Pamplona in September 1997.

At admittance (Stage 1, S1 in Table 1), the cerebral magnetic resonance imaging revealed frontoparietal tumoral lesions, predominantly left-sided and in the interhemispheric furrow with distension of the ventricular system (Fig 1A). The cytologic study revealed atypical cells in the cerebrospinal fluid. Cytogenetic analysis indicated increased chromosomal instability $(\mathrm{b} / \mathrm{c}$ index $=1.4)$ and the presence of an abnormal but nonclonal karyotype. The patient then began with chemotherapy (ICE protocol: ifosfamide, carboplatin, and etoposide) and cranial radiotherapy.

In 4 months (S2, Table 1), after four chemotherapy cycles, there was improvement in the magnetic resonance imaging with persistence of the increased signal at the ependymal level (Fig 1B) and normalization of the cytology of the cerebrospinal fluid. Nevertheless, cytogenetic studies documented an increase in genetic instability (b/c index $=1.93$ ) and in the number and complexity of the chromosomal aberrations in several nonclonal karyotypes (Table 1).

After two further cycles of chemotherapy, worsening in clinical condition was evident, with carcinomatous dissemination, thickening of the leptomeninges and the ventricular system walls (Fig 1C), and cytologic alteration of the cerebrospinal fluid. By this stage (S3, Table 1), the genetic instability had increased to 2.83 and the karyotypes manifested multiple chromosomal aberrations including the acquisition of a clonal alteration: two metaphases revealed deletion at 17p13, the locus of the TP53 tumor suppressor gene.

The patient's clinical condition continued to worsen despite aggressive treatment with chemotherapy, spinal radiotherapy, and bone marrow transplantation. The patient died 2 months later as a result of craniospinal carcinomatosis and lung metastases.

\section{DISCUSSION}

To date, neither the etiology nor the genetic pathways that lead to human meningosarcomas are clear; nevertheless, the finding of meningothelial elements within certain sarcomas has led some authors to hypothesize that the secondary sarcomatous changes in a preexisting meningioma might be considered as a meningosarcoma variant [2]. This difference is, in some instances, difficult to assess but still crucial given that the differences in both the histopathologic and genetic markers point to clinical entities which differ; specifically in terms of genomic instability which could play a role in the progression of some meningiomas [7]. 
The clinical case detailed herein was included in a protocol to evaluate the genetic instability induced by antitumoral regimens. Among other tumor types, 23 pediatric central nervous system tumors were analyzed [5]. We and others have demonstrated that these tumors do not manifest a significantly increased genetic instability at diagnosis. However, in the case of this female, both an increased sensitivity to bleomycin and an aberrant karyotype before the beginning of treatment were detected. In addition, the pathologic reports disclosed an evident deterioration between the first and second resection samples, with an increase in the number of mitoses and atypias and a clear evolution from a low-grade mesenchymal tumor to a malignant meningosarcoma. There is a lack of studies that evaluate the genomic instability in meningosarcomas, but there are several reports that relate this instability to the progression of meningiomas [7], precancerous lesions of the aerodigestive tract, or the development of relapses or second tumors of the upper aerodigestive tract.

Another significant finding in this case is that despite the initial clinical and radiologic improvement, even with normalization of the cerebrospinal fluid, there was an outstanding increase both in chromosomal fragility in bleomycin tests and in peripheral blood karyotypes, with the development of a clonal deletion (in two metaphases) of $17 \mathrm{p} 13$, the locus of the TP53 tumor suppressor gene.

The TP53 gene is a tumor suppressor gene that is frequently altered in sporadic human tumors, including those of the central nervous system. The data on the involvement of this gene in the progression or carcinogenesis of human meningeal tumors are scarce and controversial [8-10]. There is some evidence to suggest that the traditional antineoplastic treatments, namely radiotherapy and chemotherapy, may act by p53mediated apoptosis and, therefore, those cells with an altered TP53 might manifest an increased resistance to treatment-induced apoptosis. In the case reported here, the clonal deletion of $17 \mathrm{p} 13$ was coincident with a rapid worsening in clinical condition, development of cerebrospinal carcinomatosis, and multiple lung metastases which manifested no response to the most aggressive treatments.

Unfortunately, we lacked tumor tissue required for confirmation of the results obtained in peripheral blood lymphocytes given that, when the clonal deletion of TP53 developed, there was an evident clinical deterioration, and therefore, surgery was not performed. However, in the reference literature, there are reports that conclude that peripheral blood cultures of patients with different types of cancer (lung, breast, melanoma, colon, renal cell) have chromosomal breaks that are also marker chromosomes for their respective tumors [11].

In conclusion, given the aggressiveness and the poor response of this tumor to conventional treatments, it would be interesting to include a larger number of meningosarcomas in genetic instability studies to evaluate the role of genetic instability in pathogenesis and progression and in order to facilitate the optimization of individual treatment protocols.

ACKNOWLEDGMENTS: This work was partially supported by the Fondo de Investigación Cooperativa (Health Research Fund), Ministry of Health, Redes Temáticas de Investigación Cooperativa (Thematic Networks of Cooperative Research) Cáncer C10/03. The authors thank Laura Stokes for her revision of this paper. 


\section{REFERENCES}

[1] Haddad GF, Al-Mefty O. Meningeal sarcomas. In: Kaye AH, Laws ER, eds. Brain tumors: An encyclopedic approach. Toronto: Churchill Livingstone, 2001:713-24.

[2] Paulus W, Scheithauer BW. Mesenchymal, non-meningothelial tumours. In: Kleihues P, Cavenee WK, eds. Pathology and genetics of tumours of the nervous system. Lyon: IARC Press, 2000:185-9.

[3] Weber RG, Boström J, Wolter M, et al. Analysis of genomic alterations in benign, atypical and anaplastic meningiomas: Toward a genetic model of meningioma progression. Proc Natl Acad Sci USA 1997;94:14719-24.

[4] Lamszus K, Kluwe L, Matschke J, Meissner H, Laas R, Westphal M. Allelic losses at 1p, 9q, 10q, $14 q$ and $22 q$ in the progression of aggressive meningiomas and undifferentiated meningeal sarcomas. Cancer Genet Cytogenet 1999;110:103-10.

[5] Lopez de Mesa R, Lopez de Cerain Salsamendi A, Ariznabarreta LS, Calasanz Abinzano MJ, Patiño-Garcia A. Measurement and analysis of the chemotherapy-induced genetic instability in pediatric cancer patients. Mutagenesis 2002;17:171-5.

[6] Mitelman F. ISCN. An international system for human cytogenetic nomenclature. Basel: S. Karger; 1995.

[7] Simon M, Kokkino AJ, Warnick RE, Tew JM, von Demling A, Menon AG. Role of genomic instability in meningioma progression. Genes Chromosom Cancer 1996;16:265-9.

[8] Aguiar PH, Agner C, Simm R, Freitas AB, Tsanaclis AM, Plese P. p53 Protein expression in meningiomas-a clinicopathologic study of 55 patients. Neurosurg Rev 2002;25:252-7.

[9] Verheijen FM, Sprong M, Kloosterman JM, Blaauw G, Thijssen JH, Blankenstein MA. TP53 mutations in human meningiomas. Int J Biol Markers 2002;17:42-8.

[10] Kamei Y, Watanabe M, Nakayama T, Kanamaru K, Waga S, Shiraishi T. Prognostic significance of p53 and p21WAF1/CIP1 immunoreactivity and tumor micronecrosis for recurrence of meningiomas. $\mathrm{J}$ Neurooncol 2000;46:205-13.

[11] Pai SA, Cheung MC, Romsdahl MM, Multani AS, Pathak S. Can genetic instability be studied at the single chromosome level in cancer cells? Evidence from human melanoma cells. Cancer Genet Cytogenet 1999;109:51-7. 
Table 1. Cytogenetic findings at the different moments of the follow-up

\section{Bleomycin}

Stage Index* Karyotype/s

S1 $1.4 \quad 45, \mathrm{XX},-5, \operatorname{der}(11) \mathrm{t}(11 ; ?)(\mathrm{p} 15 ; ?)$

S2 $\quad 1.93 \quad 45, \mathrm{XX},+\operatorname{der}(1) \mathrm{t}(1 ; 18)(\mathrm{p} 13 ; \mathrm{q} 23),-2, \operatorname{add}(11)(\mathrm{p} 15)$, $\operatorname{der}(16) \mathrm{t}(2 ; 16)(\mathrm{q} 21 ; \mathrm{q} 24),-18$

$46, \mathrm{XX},-12, \operatorname{der}(17) \mathrm{t}(17 ; ?)(\mathrm{q} 21 ; ?)$

S3 $2.83 \quad 46, \mathrm{X}, \operatorname{add}(\mathrm{X})(\mathrm{q} 28), \operatorname{der}(2)(\mathrm{q} 24),-5, \operatorname{del}(17)(\mathrm{p} 13)$

46,XX,del(5)(p12),del(6)(q14),del(17)(p13)

* Normal Bleomycin Index $<0.8$. 

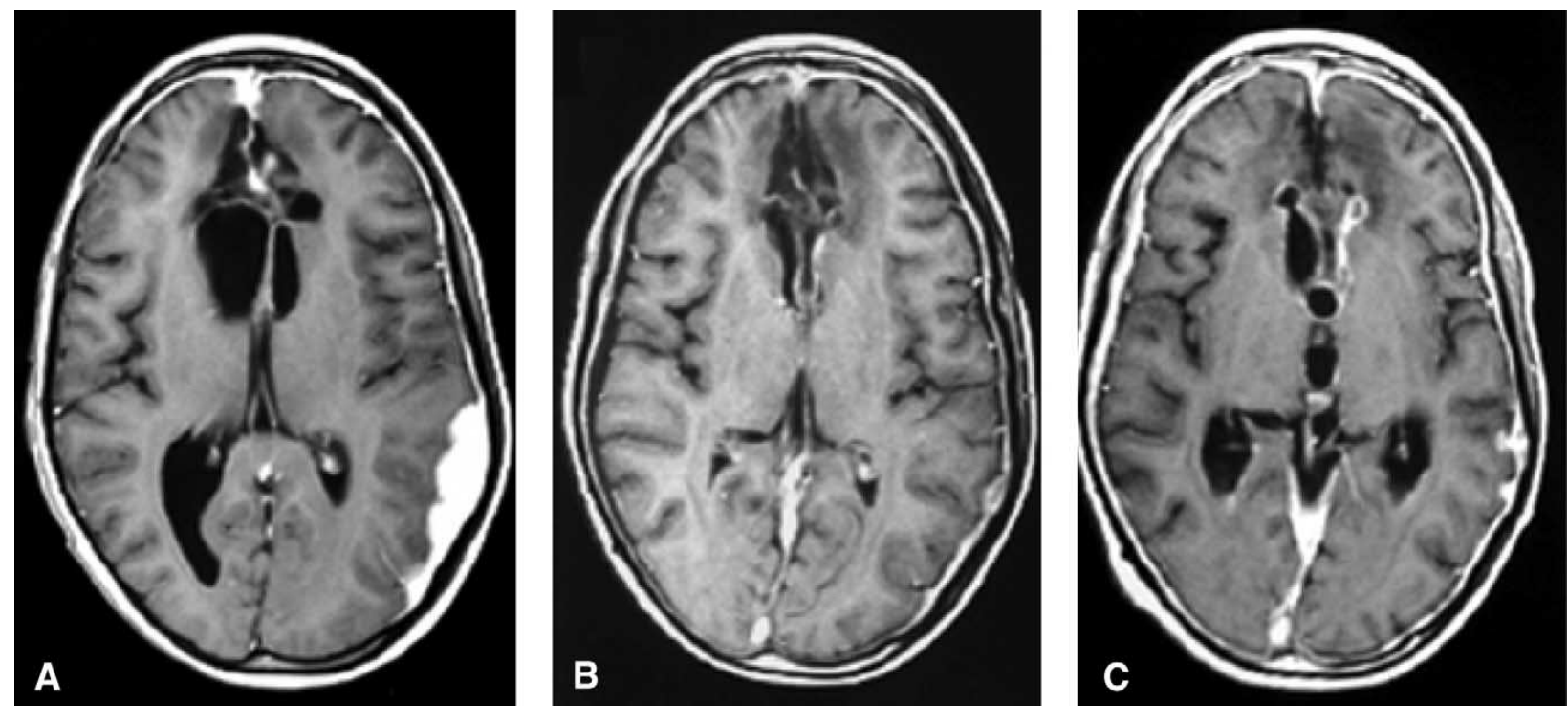

Figure 1. Magnetic resonance imaging findings at different stages of the follow-up (spin-echo $\mathrm{T}_{1}$-weighted image [TR $550 \mathrm{~ms}$, TE $14 \mathrm{~ms}$ ], paramagnetic contrast enhanced). (A) Frontoparietal tumoral lesions, predominantly left-sided and in the interhemispheric fissura with distension of the ventricular system. (B) Improvement in the magnetic resonance imaging with persistence of the hypersignal at the ependymal level. (C) Carcinomatous dissemination, thickening of the leptomeninges and the ventricular system. 